\title{
CLINICAL-HEMATOLOGICAL PROFILE OF PURE RED CELL APLASIA IN PAEDIATRICS
}

\author{
Krutika Kurhade ${ }^{1}$, Sujata Sharma ${ }^{1}$, Vaibhav Shah ${ }^{1}$, Arpita Gupta ${ }^{1}$, Neena Phade ${ }^{1}$, and \\ Ishank Goel ${ }^{1}$ \\ ${ }^{1} \mathrm{LTMMC}$
}

May 8, 2020

\begin{abstract}
Background: - The term pure red cell aplasia (PRCA) entails a group of congenital/acquired blood disorders characterized by anemia, reticulocytopenia, and insufficiency of erythroid precursors in an otherwise normo-cellular marrow. Procedures: All children diagnosed as PRCA from 2013 to 2019 at the Pediatric Hematology and Oncology Division at LTMGH, were included and their clinical profile, investigations and treatment details were analyzed. Results: Total 11 patients were diagnosed as PRCA during the study. Mean age of presentation for congenital PRCA was 8.5 months and acquired PRCA was 13.5 years. The acquired cases were patients of aplastic anemia post allogenic HSCT, with major ABO incompatibility, developing PRCA on an average of day +89 post transplant. Most common presentation was pallor in both groups. Mean hemoglobin, MCV and reticulocyte count at diagnosis was $2.7 \mathrm{gm} \%, 91.06 \mathrm{fL}, 0.2 \%$ for congenital group and $6 \mathrm{gm} \%, 128.1 \mathrm{fL}, 0.05 \%$ for acquired group. Erythroid hypoplasia was seen in bone marrow analysis of all children. In congenital group, NGS confirmed DBA in 2 children (22\%); Parvovirus was isolated in 1 child (11\%). These children were given steroid trial for a mean duration of 9 months. Four ended up on chronic transfusion program, 2 are transfusion independent, 1 is on steroid trial and 2 children were lost to follow up. Children with acquired PRCA post HSCT were treated with multiple treatment modalities with satisfactory results. Conclusion: - PRCA is a multi-faceted disease with many clinico-hematological presentations. All diagnosticpossibilities must be considered in children with a strong clinical suspicion.
\end{abstract}

\section{ABSTRACT}

Background : - The term pure red cell aplasia (PRCA) entails a group of congenital or acquired blood disorders characterized by anemia, reticulocytopenia, and insufficiency of erythroid precursors in an otherwise normo-cellular marrow.

Procedures: All children, diagnosed as PRCA from 2013 to 2019 at the Pediatric Hematology and Oncology Division at LTMGH, were included and their clinical profile, investigations and treatment details were analyzed.

Results : Total 11 patients were diagnosed as PRCA during the study period. Mean age of presentation for congenital PRCA was 8.5 months and for acquired PRCA was 13.5 years. The acquired cases were patients of aplastic anemia post allogenic HSCT, with major ABO incompatibility, developing PRCA on an average of day +89 post transplant. Most common presentation was pallor in both groups. Mean hemoglobin, MCV and reticulocyte count at diagnosis was $2.7 \mathrm{gm} \%, 91.06 \mathrm{fL}, 0.2 \%$ for congenital group and $6 \mathrm{gm} \%, 128.1 \mathrm{fL}$, $0.05 \%$ for the acquired group. Erythroid hypoplasia was seen in bone marrow analysis of all children. In the congenital group, NGS confirmed DBA in 2 children (22\%); Parvovirus was isolated in 1 child (11\%). These children were given steroid trial for a mean duration of 9 months. Four ended up on chronic transfusion program, 2 are transfusion independent, 1 is on steroid trial and 2 children were lost to follow up. Children with acquired PRCA post HSCT were treated with multiple treatment modalities with satisfactory results. 
Conclusion : - PRCA is a multi-faceted disease with many clinico-hematological presentations. All diagnostic possibilities must be considered in children with a strong clinical suspicion.

\section{INTRODUCTION-}

The term pure red cell aplasia (PRCA) entails a group of congenital or acquired blood disorders characterized by anemia, reticulocytopenia, and insufficiency of erythroid precursors in an otherwise normo-cellular marrow (1). In the congenital form most cases appear to be a result of several genetic defects. Acquired PRCA induced by parvovirus B19 infection typically produces an acute self limiting disease, called 'transient aplastic crisis' (TAC) (2). In immune-suppressed individuals or in children with underlying hemolytic anemias, Parvovirus B19 infection may result in a more chronic type of bone marrow failure, clinically apparent as PRCA.

PRCA post transplant is defined as anemia with reduced reticulocytosis and absence of red cell precursors in the bone marrow at 60 days after transplantation, it complicates about 10-20\% of all ABO-mismatched allo-stem cell transplants (3). It is more frequently observed in the constellation of group A donors in group $\mathrm{O}$ recipients and results from the presence of recipient-derived residual B lymphocytes or plasma cells which produce isohemagglutinins directed against donor RBCs (4). The risk of PRCA increases with the use of reduced intensity conditioning (5), sibling donors (6), and presence of high anti-A isohemagglutinins (7, 8). There is no known universally accepted and standardized treatment yet.

\section{METHODS}

All children diagnosed as pure red cell aplasia from 2013 to 2018 at the Pediatric Hematology and Oncology Division at LTMGH, Sion were included. Patients were diagnosed as a case of PRCA on the basis of their clinico-hematological profile and/or mutation studies. Their clinical profile, investigations and treatment details were retrospectively analyzed. A special attempt was made to elicit history of drugs, prior radiation exposure and immunization.

Criteria used for diagnosis of PRCA were anemia, reticulocytopenia and paucity of erythroid precursors in bone marrow aspirate and biopsy. The presence of giant proerythroblasts in the bone marrow portended infection with parvovirus B19 which was subsequently confirmed by serological tests. In doubtful cases, hemolytic anemias were excluded by haemoglobin-electrophoresis prior to bone marrow examination. Children were labeled to have congenital PRCA (DBA) when they had anemia at $<1$ year of age with near normal or slightly decreased neutrophil counts, variable platelet counts, reticulocytopenia, macrocytosis and normal marrow cellularity with a paucity of red cell precursors or when DBA was confirmed by Next Genration Sequencing (NGS).

The test methodology used was targeted gene sequencing in which selective capture and sequencing of the protein coding regions of the genome/genes is performed. DNA extracted from blood was used to perform targeted gene capture using a custom capture kit. The libraries were sequenced to mean $>80-100 \mathrm{X}$ coverage on Illumina sequencing platform. The sequences obtained were aligned to human reference genome (GRCh37/hg19) using BWA program $(9,10)$ and analyzed using Picard and GATK version $3.6(11,12)$ to identify variants relevant to the clinical indication. Gene annotation of the variants is performed using VEP program (13) against the Ensembl release 87 human gene model (14). Common variants were filtered based on allele frequency in 1000Genome Phase 3, ExAC, EVS, dbSNP147, 1000 Japanese Genome and our internal Indian population database.

Supportive treatment with PRBC transfusions was offered to all children. Patients with DBA were treated with prednisolone, $2 \mathrm{mg} / \mathrm{kg} /$ day in three divided doses, over a period of 4-5 weeks. Prednisolone was either gradually tapered and stopped, or continued with another immune-suppressive agent, as per the clinical response of each child.

The 2 children with acquired PRCA post HSCT were treated with multiple treatment modalities including steroids, erythropoetin, IVIG, Cyclosporine A, Bortezomib, Rituximab with satisfactory results. 
Complete response was defined as achievement of transfusion independence with hemoglobin levels $>11 \mathrm{gm} / \mathrm{dl}$.

\section{RESULTS}

Out of total 11 patients, 8 were males and 3 were females. Six were $<1$ year of age, 3 were $1-9$ years and 2 were $>9$ years. Of these, 9 were classified as congenital and 2 to have acquired PRCA. Table 1 demonstrates their clinical, hematological and investigational parameters.

Mean age of presentation for congenital PRCA was 8.5 months and for acquired PRCA was 13.5 years. Most common presenting complaint was pallor in both groups. Mean hemoglobin, MCV and reticulocyte count at diagnosis for the congenital group was $2.7 \mathrm{gm} \%$ (1.7-4.4 gm\%), 91.06 fL (76.3-112 fL), 0.2\% (0-0.96\%) respectively. While for the acquired group, it was $6 \mathrm{gm} \%$ (6.1-6 gm\%), $128.1 \mathrm{fL}$ (78.5-177.8 fL), 0.05\% (0-0.1) respectively. Erythroid hypoplasia was seen in bone marrow analysis of all children.

The two acquired cases were patients who had pure red cell aplasia post allogenic HSCT, with major ABO incompatibility. The donors in both cases were HLA matched siblings. The source of stem cells was peripheral blood in both children; and both received fludarabine-cyclophosphamide-ATG as a conditioning regime. They developed PRCA on an average of day +89 post transplant. Both the patients had AB positive donors with $\mathrm{O}$ positive recipients. Anti-donor isohematogglutinins at baseline were raised in both patients, with anti-A being higher than anti-B. Table 2 demonstrates the clinical parameters of the patients in the acquired PRCA group.

In the congenital group, next generation sequencing revealed DBA in 2 children (22\%). The abnormal genes detected were RPS19 (heterozygous missense variation in exon 3) and RPS27 (heterozygous start-loss variation in exon 1) respectively.

Parvovirus was isolated in 1 child (11\%), who was treated with IVIG at $2 \mathrm{gm} / \mathrm{kg}$ but continued to be transfusion dependent post treatment.

Children in the congenital group were given steroid trial for a mean duration of 9 months. Four ended up on chronic transfusion program, 2 are transfusion independent showing complete response, 1 is steroid dependant and 2 children were lost to follow up.

Patients with acquired PRCA post HSCT were treated with multiple treatment modalities along with cyclosporine A/ immune-suppressive agent tapering. One patient was treated with weekly erythropoietin initially followed by daily doses; IVIG at $2 \mathrm{gm} / \mathrm{kg}$, steroids (prednisolone) at $1 \mathrm{mg} / \mathrm{kg}$ with gradual tapering. Bortezomib was given for 4 doses $\left(1.4 \mathrm{~g} / \mathrm{m}^{2}\right)$. The patient ultimately responded to four once weekly doses of Rituximab (375 mg $/ \mathrm{m}^{2} /$ week). He was given supportive therapy with PRBC transfusions and GCSF during this phase. The other patient responded to Rituximab therapy. The cases developing PRCA secondary to Parvovirus infection were treated with IVIG at $2 \mathrm{gm} / \mathrm{kg}$.

\section{DISCUSSION}

Pure red cell aplasia (PRCA) is a syndrome defined by a normocytic normochromic anemia with severe reticulocytopenia and marked reduction or absence of erythroid precursors from the bone marrow. All the children in this study had reticulocytopenia $(<1 \%)$ and erythroid hypoplasia was seen in bone marrow analysis of all children. Diamond-Blackfan anemia is a congenital form of PRCA. Acquired PRCA may be either a primary disorder or secondary to some other disorder or agent. DBA is typically treated with glucocorticoids, usually prednisone. Hematopoietic stem cell transplantation has also been used in patients unresponsive to glucocorticoids at sustainable doses (1).

Immunosuppressive therapy in PRCA includes steroids, cytotoxic agents, cyclosporine A, anti-thymocyte globulin. Traditional immunosuppression with oral corticosteroids, usually prednisone, in dose of $1 \mathrm{mg} / \mathrm{kg}$ appears to have a $40 \%$ response rate. Prednisone is tapered after a response is obtained, or decreased and used in association with another agent if remission is not obtained (1). In the present study, 2 children were able to achieve and maintain a complete response after completion of steroid therapy. Four children ended 
up on chronic transfusion program despite immunosuppresive therapy, 1 child is currently on steroid trial and 2 children were lost to follow up.

Pure red cell aplasia (PRCA) is a severe consequence of major and bi-directional ABO-mismatched allogeneic stem cell transplantation (allo-SCT), likely the result of persistent isoagglutinin-producing host plasma cells that have escaped pre-transplant conditioning or graft vs. plasma cell effect (15) It occurs in up to $29 \%$ of patients with a major ABO-mismatched donor. Graft-mediated effects against recipient hematopoiesis in the absence of effects against recipient isohemagglutinin producing cell populations may result in delayed donor RBC chimerism and PRCA owing to loss of recipient erythropoiesis and concomitant inhibition of donor erythropoiesis (5). The recipient isohemagglutinins disrupt normal bone marrow maturation of medullary precursors at the colony-forming units-erythroid stage, which is the earliest point of $\mathrm{ABO}$ antigen presentation (16).

In such cases various treatment modalities to remove persisting isohemagglutinins have been described which may include erythropoietin, plasma exchange, taper of immunosuppressive drugs, and administration of donor leukocyte infusions. The use of rituximab, a monoclonal antibody directed against CD20-positive B cells, has been shown to be effective in some case reports (4).

PRCA after ABO-incompatible bone marrow or stem cell transplant is observed most commonly with the combination of a blood group A donor and a blood group $\mathrm{O}$ recipient (1). In both of the acquired group patients, the recipient blood group was $\mathrm{O}$ positive and donor blood group was $\mathrm{AB}$ positive. Anti-donor isohematogglutinins at baseline were raised in both patients, with anti-A being higher than anti-B. Patients with anti-A isoagglutinins against donor RBC have been found to develop PRCA more frequently than patients with anti-B (17). In one of the patients, anti-A titres persisted longer than those of anti-B. These observations have been explained by higher baseline levels of anti-A versus anti-B isohemagglutinins, increased cell surface density of A compared with $\mathrm{B}$ antigens on the RBC membrane, or increased complement-fixing capacity of red-cell-bound anti-A compared with anti-B (5).

Cyclosporine A has potent immunosuppressive effects on $\mathrm{T}$ cells, but minimal to no direct effects on B cells. Discontinuation of Cyclosporine A induces a graft-mediated immune effect against host cells involved in isohemagglutinin production, leading to resolution of PRCA in some cases (5); but this was not sufficient in our patients. Both the children were given erythropoietin, Rituximab. One child required treatment with IVIG, prednisolone, Bortezomib before finally responding to Rituximab. Both cases ultimately achieved complete response.

In conclusion, owing to the increased frequency of reduced intensity conditioning in $\mathrm{ABO}$ incompatible settings, more studies are indicated to follow the trend, etiology, preventative measures and treatment strategies of acquired PRCA post-transplant. Similarly, in congenital PRCA, further treatment avenues for children who remain transfusion dependant post adequate therapy need to be explored.

CONFLICT OF INTERESTS-

None

ACKNOWLEDGEMENT-

REFERENCES-

1. Means RT Jr. Pure red cell aplasia. Hematology Am Soc Hematol Educ Program . 2016;2016(1):51-56. doi:10.1182/asheducation-2016.1.51

2. Fisch P, Handgretinger R, Schaefer H. Pure red cell aplasia. Br J Haematol . 2000;111(4):1010-1022. doi:10.1046/j.1365-2141.2000.02429.x

3. Karp R, Arnason J, Rosenblatt J et al. Pure Red Cell Aplasia after ABO-Mismatched Allogeneic Stem Cell Transplantation Treated with Therapeutic Plasma Exchange and Rituximab. Blood . 2015;126(23):5453. 
4. Worel N. ABO-Mismatched Allogeneic Hematopoietic Stem Cell Transplantation. Transfusion Medicine and Hemotherapy . 2016;43(1):3-12. doi:10.1159/000441507

5. Bolan CD, Leitman SF, Griffith LM et al. Delayed donor red cell chimerism and pure red cell aplasia following major ABO-incompatible nonmyeloablative hematopoietic stem cell transplantation. Blood 2001;98(6):1687-1694

6. Mielcarek M, Leisenring W, Torok-Storb B et al. Graft-versus-host disease and donor-directed hemagglutinin titers after ABO-mismatched related and unrelated marrow allografts: evidence for a graftversus-plasma cell effect. Blood 2000;96(3):1150-1156.

7. Lee JH, Lee KH, Kim S, Lee JS, Kim SH et al. Anti-A isoagglutinin as a risk factor for the development of pure red cell aplasia after major ABO-incompatible allogeneic bone marrow transplantation. Bone Marrow Transplant 2000;25(2):179-184.

8. Schetelig J, Breitschaft A, Kroger N et al. Cooperative Transplantations Study Group: After major ABO-mismatched allogeneic hematopoietic progenitor cell transplantation, erythroid engraftment occurs later in patients with donor blood group A than donor blood group B. Transfusion 2005;45(5):779787

9. Li, H. and R. Durbin. Fast and accurate long-read alignment with Burrows-Wheeler transform. Bioinformatics, 2010. 26(5): p. 589-95.

10. Meyer, L.R., et al ., The UCSC Genome Browser database: extensions and updates 2013. Nucleic Acids Res, 2013. 41(D1): p. D64-9.

11. McKenna, A., et al ., The Genome Analysis Toolkit: a MapReduce framework for analyzing nextgeneration DNA sequencing data. Genome Res, 2010. 20(9): p. 1297-303.

12. Li, H., et al ., The Sequence Alignment/Map format and SAMtools. Bioinformatics, 2009. 25(16): p. 2078-9.

13. McLaren, W., et al ., Deriving the consequences of genomic variants with the Ensembl API and SNP Effect Predictor. Bioinformatics, 2010. 26(16): p. 2069-70.

14. ENSEMBL: http://www.ensembl.org

15. Aung F, Lichtiger B, Rondon G et al. Pure Red Cell Aplasia in Major ABO-Mismatched Allogeneic Hematopoietic Stem Cell Transplantation Is Associated with Severe Pancytopenia. Biology of Blood and Marrow Transplantation . 2016;22(5):961-965. doi:10.1016/j.bbmt.2016.02.008

16. Barge A, Johnson G, Witherspoon R, Torok-Storb B. Antibody-mediated marrow failure after allogeneic bone marrow transplantation. Blood . 1989;74(5):1477-1480. doi:10.1182/blood.v74.5.1477.bloodjournal7451477

17. Lee J, Lee K, Kim S et al. Anti-A isoagglutinin as a risk factor for the development of pure red cell aplasia after major ABO-incompatible allogeneic bone marrow transplantation. Bone Marrow Transplant . 2000;25(2):179-184. doi:10.1038/sj.bmt.1702121

\section{LEGEND-}

\section{Hosted file}

Table_1.docx available at https://authorea.com/users/319624/articles/449334-clinicalhematological-profile-of-pure-red-cell-aplasia-in-paediatrics

\section{Hosted file}

Table 2.docx available at https://authorea.com/users/319624/articles/449334-clinicalhematological-profile-of-pure-red-cell-aplasia-in-paediatrics 